\title{
A Fabry-Perot refractometer for chemical vapor sensing by solid-phase microextraction
}

\author{
R. St-Gelais ${ }^{1}$, G. Mackey ${ }^{2}$, J. Saunders ${ }^{2}$, J. Zhou ${ }^{2}$, A. Leblanc-Hotte ${ }^{1}$, A. Poulin ${ }^{1}$, J.A. Barnes ${ }^{2}$, \\ H.-P. Loock ${ }^{2}$, R. S. Brown ${ }^{2}$, Y.-A. Peter ${ }^{1}$ \\ ${ }^{1}$ Department of Engineering Physics, Ecole Polytechnique de Montréal, Montréal, QC, H3C 3A7, Canada \\ ${ }^{2}$ Department of Chemistry, Queen's University, Kingston, ON, K7L 3N6, Canada
}

\begin{abstract}
The contour lithography method [1] is used to improve the fabrication yield of previously demonstrated [2] microfluidic Fabry-Perot (FP) refractive index (RI) sensors. The sensors are then coated with polydimethylsiloxane (PDMS) based polymers to detect vapor analytes by solid-phase microextraction (SPME). Preliminary characterization of devices coated with two different polymers and exposed to xylenes vapors yields a maximum sensitivity of $0.015 \mathrm{~nm} / \mathrm{ppm}$ and a detection limit below $120 \mathrm{ppm}$.
\end{abstract}

Keywords-Chemical sensors; Fabry-Perot; Microfluidics; Microoptics; Optical sensors; Optical device fabrication

\section{INTRODUCTION}

PDMS based polymers have the ability to absorb and to concentrate chemical vapors by SPME. Upon absorption, the polymer RI changes proportionally to the concentration of the targeted gas in its vicinity, the RI difference between the polymer and the gas, and the SPME extraction efficiency. Therefore, monitoring the RI of siloxane polymers allows the detection and the differentiation of, for instance, volatile organic compounds, in real time, at the detection site.

This was previously demonstrated with long-period fiber Bragg gratings (LPFBG) and with silicon-on-insulator (SOI) Mach-Zehnder RI sensors [3, 4]. These sensors are based on the propagation of an evanescent light wave within a thin surface layer at the polymer-sensor interface. This sometimes complicates the chemical quantification since surface effects occurring at the interface can alter the sensor performance. Moreover, for LPFBGs, the initial RI of the polymer needs to be precisely matched to that of the cladding in order to obtain sufficient penetration depth of light in the polymer. The MachZehnder sensors do not have the RI matching requirement, but are still sensitive to effects at the waveguide-coating interface. They also require coupling into nanoscale structures.
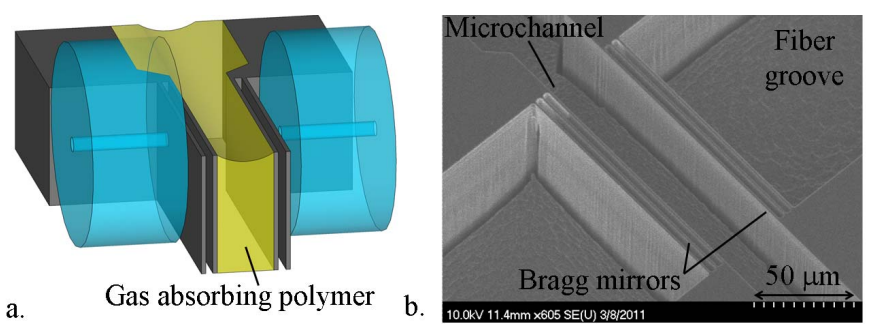

Figure 1. (a) Schematic representation of a two silicon layers FP sensor filled with the SPME polymer and interfaced with single mode optical fibers. (b) Scanning electron micrograph of a three silicon layers FP sensor (before polymer coating).
For these reasons, the use of the FP RI sensor previously reported in [2] and presented in Fig. 1 is proposed. This sensor has integrated fiber alignment grooves and performs bulk RI sensing, which facilitates optical fiber alignment and polymer coating. An improved fabrication process is presented in section II. Optical characterization of FPs having two (Fig. 1 a) and three (Fig. $1 \mathrm{~b}$ ) silicon ( $\mathrm{Si}$ ) layers per mirror is presented in section III, together with preliminary SPME experiments.

\section{DESIGN AND FABRICATION}

The original fabrication process [2] of the FP sensor involved a single photolithography step followed by a single deep reactive ion etching (DRIE) of the multilayer mirrors, the microfluidic system and the alignment grooves. This process allowed the fabrication of working devices, but the yield was low since the final thickness of each layer of the mirrors was difficult to predict. Silicon layers facing the large openings (grooves and microchannel) suffered from less diffraction during photolithography but more undercut during DRIE. Conversely, the inner layers of the mirrors experienced more diffraction and less undercut. This resulted in unpredictable optical characteristics that prevented successful transfer of large numbers of sensors to the chemistry lab for parallel testing of various polymers and gases.
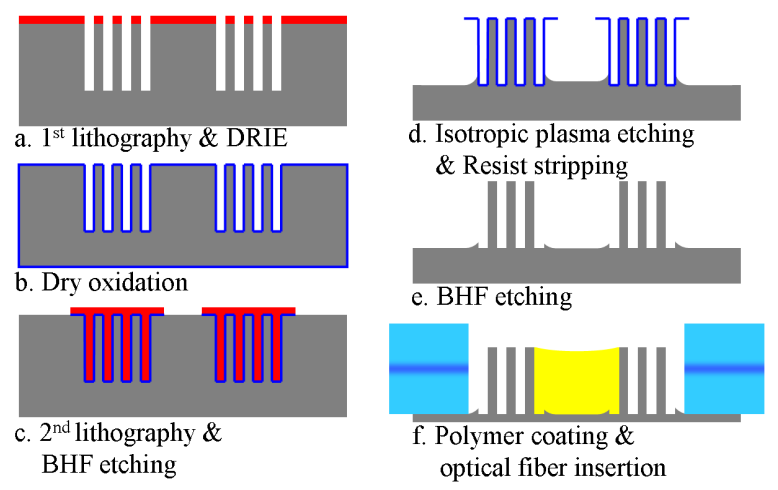

Figure 2. Schematic cross section of the fabrication process for a three silicon layers FP sensor.

In order to obtain more predictable characteristics, new sensors were fabricated by the two mask contour lithography process proposed in [1] and illustrated in Fig. 2. The mirrors and the contours of the larger openings (grooves and microfluidics) are first etched by DRIE (step a). After a dry oxidation step (b) to protect the mirrors and the contours, a second mask defines openings within the contours of the larger 
features (step c). These larger features are then isotropically etched in a $\mathrm{SF}_{6}$ plasma (step d) and the remaining oxide is removed (step e) in a buffered hydrofluoric acid solution (BHF). A drop of liquid polymer is finally placed in a $1 \mathrm{~mm}$ wide reservoir that connects to the microchannel (Fig. 1). The polymer flows between the mirrors by capillary action and then cures at room temperature for a few hours.

Using this fabrication process, the Si-air period of the mirrors remains constant throughout all the fabrication steps. The final thickness of each layer is unknown, but all layers of a same material ( $\mathrm{Si}$ or air) have the same thickness. This leaves only one unknown to consider during mask design and therefore allows predictable optical characteristics.

\section{RESULTS}

Figure 3 (a) presents the transmission spectrum, before coating, of FPs having two (Fig. 1 a) and three (Fig. 1 b) Si layers per mirror. The thicknesses of the $\mathrm{Si}$ and air layers are respectively of $11 \lambda_{0} / 4 n_{S i}$ and $9 \lambda_{0} / 4 n_{A i r}$, where $n$ is the RI of the layer. This ensures a broadband high reflectivity at the central wavelength of the measurement setup $\left(\lambda_{0}=1550 \mathrm{~nm}\right)$. As predicted by the model-which takes into account Gaussian beam divergence and $25 \mathrm{~nm}$ rms of surface roughness at the silicon-air interfaces [5] - FP peaks are clearly visible between $1500 \mathrm{~nm}$ and $1600 \mathrm{~nm}$. As the RI changes between the mirrors, these peaks are expected to shift with a sensitivity of $10^{3} \mathrm{~nm} / \mathrm{RI}$ unit. FPs based on three Si layers per mirror allow slightly narrower resonance peaks (e.g.: at $\lambda=1580 \mathrm{~nm}$ ) at the expense of important signal loss $(>10 \mathrm{~dB})$. Therefore, FPs based on two Si layers are preferred for the following SPME experiments.
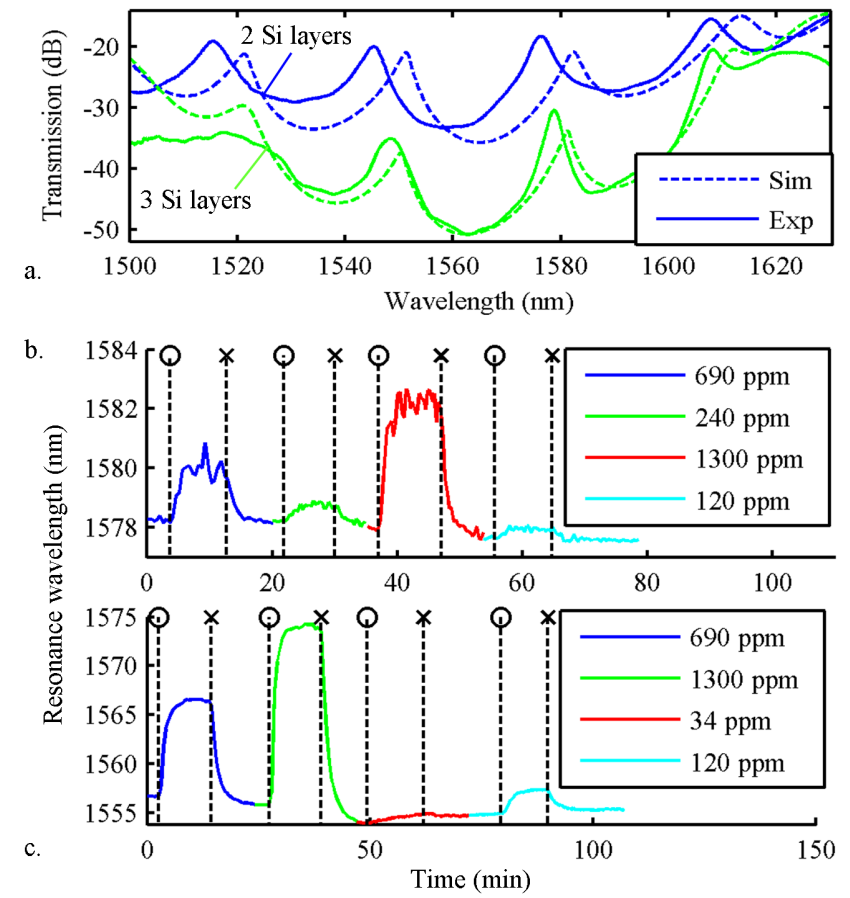

Figure 3. (a) Experimental and simulated transmission spectrum, before polymer coating, of FPs having two and three silicon layers per mirror. (b, c) Resonance wavelength of two layers FPs coated with commercial PDMS (b) and with a PDMS-PDPS copolymer (c). O and X symbols designate the beginning and the end of exposition to the labeled dilutions of xylenes vapor.
Two FP cavities are coated with siloxane polymers and are exposed to various dilutions of xylenes vapor in nitrogen. The center wavelength of a resonance peak is measured every 20 seconds with a tunable laser. The first device is coated with commercial SYLGARD ${ }^{\circledR} 184$ PDMS. Its response to xylenes is presented in Figure 3 (b) and yields a sensitivity of $3 \times 10^{-3}$ $\mathrm{nm} / \mathrm{ppm}$. The second device (Fig. 3 c) is coated with a 9:1 PDMS-polydiphenylsiloxane (PDPS) copolymer and yields a fivefold increase of the sensitivity to xylenes $\left(1.5 \times 10^{-2}\right.$ $\mathrm{nm} / \mathrm{ppm})$. Its response to a concentration of $34 \mathrm{ppm}$ is somewhat visible but seems distorted by the liberation of the previous 1300 ppm gas uptake (possibly because of some contraction of the polymer during desorption). However, the response to $120 \mathrm{ppm}$ is clearly visible, which indicates a detection limit below 120 ppm.

The RI difference between xylenes $(n \approx 1.48)$ and commercial PDMS $(\mathrm{n} \approx 1.40)$ is larger than between xylene and the copolymer $(n \approx 1.42)$ [3]. Therefore, the higher sensitivity obtained with the copolymer is not due to a larger RI difference but to a larger SPME partition coefficient.

\section{CONCLUSION}

FP refractive index sensors yielding predictable optical characteristics were successfully fabricated by contour lithography. Coating them with gas absorbing polymers allowed the detection of xylenes vapor by SPME with a detection limit below $120 \mathrm{ppm}$. The devices therefore have potential to outperform sensors based on LPFBG [3] and SOI interferometers [4], in addition to simplifying the packaging and the polymer coating process. Future work will involve testing of other siloxane polymers and other gases over a wider range of concentrations, in order to determine more precisely the detection limit and to evaluate the detection specificity.

\section{REFERENCES}

[1] Y. Mita, M. Kubota, T. Harada, F. Marty, B. Saadany, T. Bourouina and T. Shibata, "Contour lithography methods for DRIE fabrication of nanometre-millimetre-scale coexisting microsystems," J. Micromech. Microeng., vol. 16, pp. S135-S141, 2006.

[2] R. St-Gelais, J. Masson, Y.-A. Peter, “All-silicon integrated Fabry-Pérot cavity for volume refractive index measurement in microfluidic systems,” Appl. Phys. Lett., vol. 94, p. 243905, 2009.

[3] J.A. Barnes, M. Dreher, K. Plett, R.S. Brown, C.M. Crudden and H.-P. Loock, "Chemical sensor based on a long-period fibre grating modified by a functionalized polydimethylsiloxane coating," Analyst, vol 133, pp. 1541-1549, 2008.

[4] J. Saunders, M.A. Dreher, J.A. Barnes, C.M. Crudden, J. Du, H.-P. Loock, D.-X. Xu, A. Densmore, R. Ma, S. Janz, M. Vachon, J. Lapointe, A. Delâge, J. Schmid and P. Cheben, "Detection of lead contamination of water and VOC contamination of air using SOI micro-optical devices," Proc. IEEE 7th international conference on group IV photonics, Beijing, 2010.

[5] R. St-Gelais, A. Poulin and Y.-A. Peter, "Effects of divergence and surface roughness in in-plane deeply etched multilayer resonators," in preparation. 